\title{
Síndrome de Down: etiologia, caracterização e impacto na família
}

\author{
Nara Liana Pereira Silva \\ Maria Auxiliadora Dessen \\ Universidade de Brasília
}

\begin{abstract}
Resumo
A síndrome de Down é uma desordem genética que causa deficiência mental em graus variados. Atualmente, seu quadro clínico é bastante conhecido, sendo as características fenotípicas essenciais para o diagnóstico precoce da síndrome. A inserção adequada da criança no seu contexto sociocultural é de grande importância para a sua adaptação e bem-estar, e a família desempenha um papel primordial como mediadora desse processo. Neste artigo, discutimos questões relativas à caracterização e etiologia da síndrome de Down, destacando a participação da família no processo de desenvolvimento e adaptação dessa criança. Acreditamos que o conhecimento das alterações provocadas pela síndrome de Down constitua o primeiro passo para a implementação de programas voltados para uma melhor atuação de profissionais e pais.
\end{abstract}

Palavras-chave: síndrome de Down; etiologia; família.

\begin{abstract}
Down Syndrome: etiology, characteristics, and its effects on family

Down syndrome is a genetic disorder that causes mental retardation in varying degrees. At the present time, the symptoms of this syndrome are well known, being the babies' phenotypical characteristics a very essential factor to the early diagnosis of this disorder. The child being introduced in the sociocultural context contributes to his/her adaptation and well-being. In these cases, the family plays an important role as a mediator between the child and the environment. Therefore, this article aims to describe some aspects of Down syndrome's characteristics and its etiology, emphasising the family participation in the development and adaptation process of Down syndrome children. We believe that the scientific knowledge of alterations caused by this kind of disorder constitutes the first step to implement effective programmes for parents and professionals.
\end{abstract}

Keywords: Down syndrome; etiology; family.

Neste artigo, apresentamos, primeiramente, um breve histórico sobre a síndrome de Down (SD) e descrevemos, resumidamente, o quadro clínico dessa desordem genética. Os fatores que contribuem para a incidência da síndrome e um panorama geral sobre algumas pesquisas a respeito das características de comportamento, temperamento e preferências de pessoas com SD são abordados nos tópicos seguintes. $\mathrm{Na}$ parte final, é dada ênfase à importância da família na promoção do desenvolvimento da criança com síndrome de Down, e algumas considerações finais são destacadas, particularmente no que se refere à elaboração e implementação de programas de educação familiar e de intervenção.

\section{A síndrome de Down: uma visão geral}

\section{Um breve histórico}

A síndrome de Down (SD) tem registros antigos na história do homem, sendo os primeiros trabalhos científicos datados do século XIX. Contudo, a história da humanidade mostra crianças com SD sendo retratadas, principalmente, por pintores como Andrea Mantegna (1431-1506) e Jacobs Jordaens (1539-1678). Em 1838, Esquirol fez referência à síndrome em um dicionário médico. Outros registros são constatados na história, por exemplo, no livro de Chambers, datado de 1844, no qual a síndrome de Down é denominada "idiotia do tipo mongolóide", e na descrição feita por Edouard Seguin (entre 1846 e 1866) que se referia à síndrome como um subtipo de cretinismo classificado como "cretinismo furfuráceo" (Schwartz-man, 1999a). Entretanto, o reconhecimento da síndrome de Down 
como uma manifestação clínica só ocorreu com o trabalho de Langdon Down, em 1866, que foi influenciado pelos conceitos evolucionistas da época. Em seu trabalho, Langdon Down afirmava a existência de raças superiores a outras, sendo a deficiência mental característica das raças inferiores. Além disso, a tuberculose, presente nos genitores de crianças com SD daquela época, era considerada como um fator etiológico.

Depois desse trabalho inicial, vieram outros que contribuíram para aprofundar o conhecimento sobre a síndrome de Down. Dentre eles, os trabalhos de Fraser e Michell (1876), o de Ireland (1877), que distinguiu a "idiotia mongolóide" da "idiotia cretinóide", o de Wilmarth (1890) e o de Telford Smith, em 1896, que descobriu uma técnica de tratamento para estas crianças utilizando o hormônio tireoidiano. Mas, foi somente em 1932, que um oftalmologista holandês chamado Waardenburg sugeriu que a ocorrência da síndrome de Down fosse causada por uma aberração cromossômica. Dois anos mais tarde, em 1934, nos Estados Unidos, Adrian Bleyer supôs que essa aberração poderia ser uma trissomia. Parecia, portanto, que a descoberta da causa da síndrome de Down estava próxima. Contudo, foram necessárias mais de duas décadas para que isto ocorresse. Foi somente em 1959 que o Dr. Jerome Lejeune e Patricia A. Jacobs e seus respectivos colaboradores descobriram, quase que simultaneamente, a existência de um cromossomo extra (Schwartzman, 1999a).

A denominação de síndrome de Down só foi proposta após várias outras denominações terem sido usadas: imbecilidade mongolóide, idiotia mongolóide, cretinismo furfuráceo, acromicria congênita, criança mal-acabada, criança inacabada, dentre outras. Obviamente, alguns desses termos apresentam um alto grau pejorativo, incluindo o termo mongolismo, que foi amplamente utilizado até 1961, quando as críticas contrárias ao seu uso despontaram. Em decorrência disso, segundo Schwartzman (1999a), esta terminologia foi suprimida nas publicações da Organização Mundial de Saúde (OMS), a partir de 1965, prevalecendo a denominação de síndrome de Down, embora o termo mongolismo ainda seja utilizado na linguagem cotidiana.

\section{Caracterização}

Desde 1959, quando foi comprovada a existência de um cromossomo extra na constituição cromossômica dos indivíduos com SD, várias foram as descobertas sobre o seu quadro clínico, que é bastante conhecido, hoje. A síndrome de Down, portanto, é "uma cromossomopatia, ou seja, uma doença cujo quadro clínico global é explicado por um desequilíbrio na constituição cromossômica (no caso, a presença de um cromossomo 21 extra), caracterizando, assim, uma trissomia simples" (Brunoni, 1999, p. 32). Segundo Schwartzman (1999b), o cariótipo 47, XX, + 21 ou $47, \mathrm{XY},+21$ está presente em cerca de $95 \%$ dos casos da composição cromossômica das pessoas com síndrome de Down.

Esta síndrome também pode ser caracterizada por uma translocação ou um mosaico (Schwartzman, 1999b). Na translocação, o cromossomo 21 adicional está fundido a um outro autossomo; a mais comum é aquela existente entre os cromossomos 14 e 21. A ocorrência deste tipo de anomalia se dá em 5\% dos casos diagnosticados, embora Brunoni (1999) afirme que a incidência seja de 1,5 a $3 \%$. Já a síndrome de Down caracterizada por um mosaico representa um grupo menor, no qual as células trissômicas aparecem ao lado de células normais.

Dentre as características fenotípicas desta síndrome destacam-se a braquicefalia, descrita por um diâmetro fronto-occipital muito pequeno, fissuras palpebrais com inclinação superior, pregas epicânticas, base nasal achatada e hipoplasia da região mediana da face. Além dessas características da face, observa-se, também, que o pescoço é curto, podendo estar presente apenas uma prega palmar; a pina é pequena e displásica; a língua é protusa e hipotônica; há clinodactilia do $5^{\circ}$ dedo das mãos e uma distância aumentada entre o $1^{\circ}$ e o $2^{\circ}$ dedos dos pés. Em geral, as crianças com SD apresentam hipotonia muscular e são muito sonolentas. Logo após o nascimento, elas mostram dificuldades para a sucção e deglutição. Observa-se, também, um atraso no desenvolvimento de alguns reflexos do bebê, havendo um comprometimento na postura de semiflexão dos quadris, que pode não ser evidente ou, até mesmo, estar ausente.

A linguagem dessas crianças é bastante comprometida, principalmente se comparadas com o grupo de crianças com desenvolvimento 'normal'. Segundo Tristão e Feitosa (1998), o quadro apresentado por essas crianças pode ser mais complexo, uma vez que associado ao atraso no desenvolvimento da linguagem, encontra-se também uma "...instabilidade na produção vocal, organização gramatical pobre, fala funcional quando adquirida na maioria dos casos" (p. 135). Mas, segundo estas autoras, alguns indivíduos podem atingir altos níveis de linguagem. De acordo com Schwartzman (1999c), apesar destas dificuldades, “...a maioria dos indivíduos faz uso funcional da linguagem e compreende as regras utilizadas nas conversações" (p. 62).

A deficiência mental é uma das características mais presentes na síndrome de Down devido, provavelmente, a um atraso global no desenvolvimento, que varia de criança para criança. Embora o QI dessas crianças seja classificado como abaixo da média, os 
pesquisadores e profissionais têm enfatizado a necessidade de se discutir mais sobre as habilidades das crianças deficientes mentais para a realização das atividades de vida diária, tais como andar, vestir-se, alimentar-se com independência, aprender a ler etc., ao invés de destacá-lo como uma medida importante do grau de comprometimento.

\section{Fatores que contribuem para a incidência da SD}

Pouco se conhece a respeito das causas que levam ao nascimento de crianças com síndrome de Down. Para Schwartzman (1999b), alguns fatores endógenos e exógenos contribuem para a maior ou menor incidência da desordem. Um dos fatores endógenos mais freqüentemente associados a esta síndrome é a idade da mãe, pois as mulheres já nascem com uma quantidade de óvulos que envelhecem à medida que elas também envelhecem. Portanto, quanto mais velha a mãe, maior será a probabilidade de incidência da SD. De acordo com a tabela elaborada pelo ECLAMC Estudo Colaborativo Latino-Americano de Malformações Congênitas (conforme citado por Brunoni, 1999), o risco de uma mulher de 34 anos, por exemplo, gerar uma criança com síndrome de Down é de um nascimento para cada 392 mães com 34 anos (1/392), e para uma mãe de 40 anos, o risco é de 1/80. No estudo de Pereira-Silva (2000), com seis famílias de crianças com SD, quatro mães tinham idades entre 36 e 43 anos. É preciso lembrar que estes dados não descartam a possibilidade de incidência da desordem em crianças com mães mais jovens.

Schwartzman (1999b) inclui a ausência de diagnóstico pré-natal e a exposição à radiação como fatores que contribuiriam para a incidência da síndrome de Down. Com relação ao diagnóstico, atualmente existem exames (amniocentese, coleta de vilo corial, exame de sangue, ultra-som) que podem detectar as condições do feto, incluindo a ocorrência da SD. Segundo Schwartzman, se esses exames fossem realizados como rotina, poderiam ser identificadas $60 \%$ das gestações afetadas. Este autor ressalta a necessidade da ampliação e da redução de custos com os exames para que se possa atingir uma maior parcela da população. A influência da exposição à radiação foi particularmente constatada através de uma investigação realizada em uma região da Inglaterra em que, durante um período de tempo, as pessoas ficaram expostas à radiação ionizante devido a testes nucleares. Um aumento significativo da SD foi detectado entre as crianças nascidas naquele período compreendido pelos testes atômicos. Uma outra possibilidade que tem sido apontada como contribuindo para o aumento da incidência da SD é o uso de pílulas anticoncepcionais; porém, não há comprovação a esse respeito (Schwartzman, 1999b).

\section{O que as pesquisas contam a respeito da SD?}

Segundo Brunoni (1999), atualmente, as crianças com síndrome de Down têm apresentado uma sobrevida maior devido, em parte, aos avanços alcançados e propiciados a elas por tratamentos diversos, como nas cardiopatias congênitas, responsáveis por grande parte das mortes. Associadas ou não às cardiopatias estão também incluídas as diversas infecções respiratórias e outras malformações congênitas e até a leucemia, em grau menor. Em três pesquisas, totalizando 88 crianças e 41 adultos com SD e suas respectivas famílias, Carr (1994) encontrou que a grande maioria da amostra apresentava boa saúde, embora tivessem sido diagnosticados problemas de pele, epilepsia (em menos de $15 \%$ das pessoas) e desordens cardíacas (de $20 \%$ a $40 \%$ das pessoas). A incidência de câncer foi similar à taxa da população geral, com exceção do alto risco de leucemia na infância.

A associação entre a doença de Alzheimer e a SD é reconhecida por mais de um século. Os sinais neuropatológicos dessa doença têm sido encontrados nos cérebros de todas as pessoas com SD acima de 40 anos e, até mesmo naquelas com 35, 30 ou 20 anos de idade (Carr, 1994). Parece não haver dúvida de que a doença de Alzheimer ocorra mais precocemente nessas pessoas que na população geral. Quanto às desordens psiquiátricas, por exemplo, neuroses, desordens de conduta e esquizofrenias, as pessoas com SD parecem ser menos vulneráveis a elas que outros indivíduos com deficiências de aprendizagem. No entanto, a depressão foi mais freqüente $(10 \%)$ que nos indivíduos com outros problemas de aprendizagem.

Com relação ao quociente de inteligência (QI) de crianças com SD, Carr (1994) observou um declínio à medida que a idade de sua amostra avançava. Contudo, $2 / 3$ dos jovens foram considerados independentes nas áreas de alimentação e toalete, de $1 / 3$ a $2 / 3 \mathrm{em}$ vestir-se, a metade em tomar banho e $1 / 4$ em lavar os cabelos. Nove rapazes, em um grupo de 22, eram capazes de barbear-se sem ajuda e 12 moças, em um grupo de 19, podiam enfrentar sozinhas os cuidados com a menstruação. Pouco mais da metade das pessoas com 21 anos eram independentes em todas as quatro áreas: alimentação, vestir-se, lavagem dos cabelos e toalete. Esses dados comprovam que essas pessoas podem tornar-se independentes ao longo da vida, apesar de apresentarem um desenvolvimento mais lento e de haver diferenças de criança para criança.

Com relação às características comportamentais e de temperamento de crianças que apresentam deficiência mental, a literatura é relativamente vasta, com grande parte dos estudos dedicando-se aos problemas de comportamento apresentados por essas crianças (Cuskelly \& Dadds, 1992; Leary \& Verth, 1995; Le- 
manek, Stone \& Fishel, 1993; Turnbull \& Ruef, 1996). Estes são, em geral, diversificados e dependem do tipo de deficiência e do grau de comprometimento da criança. Por exemplo, as crianças com síndrome de Down, no estudo de Cuskelly e Dadds, apresentaram mais problemas de comportamento do que seus irmãos, destacando-se os problemas de atenção e imaturidade. Já, Gibson (citado em Ganiban, Wagner \& Cicchetti, 1990) relata que as crianças com SD apresentam o estereótipo de obstinadas, mas também são afetivas e de temperamento fácil. Este autor afirma que, embora a maioria das crianças e adultos tenham se adequado a este estereótipo, há subgrupos de indivíduos com SD que apresentam-se agressivos, agitados e difíceis de manejar, o que demonstra que o temperamento dos indivíduos com SD não é uniforme. Nos estudos de Carr (1994), a maioria dos genitores caracterizou seus filhos como amáveis, afetuosos e atenciosos e, no estudo de Pereira-Silva (2000), as crianças pré-escolares foram descritas como sendo birrentas, calmas ou agitadas e, também, irritadas.

De acordo com Carr (1994), no tocante às atividades preferidas dos indivíduos com SD, destacam-se, em ambiente doméstico: assistir à TV, ouvir música, desenhar, colorir e olhar livros. Os mais variados tipos de esportes foram mencionados como atividades preferidas fora de casa, estando a metade dos jovens da amostra engajados regularmente na natação. Pelo menos $1 / 5$ dos jovens faziam um tipo de esporte, e 2/3, dois ou mais tipos. Esses jovens participavam de excursões e também viajavam. Apesar de alguns freqüentarem clubes destinados a pessoas com deficiências, poucos se engajavam em eventos abertos a toda a comunidade.

Com relação aos contatos com o sexo oposto, há poucos estudos que se dedicam a este tema. Nos relatos de Carr (1994), 17\% dos adolescentes tinham um namorado e $15 \%$ já tinham tido namorado no passado. Um terço dos adolescentes com 21 anos desejavam casar. Contudo, os relacionamentos 'sérios' eram desencorajados pelos genitores e, também, pela equipe de profissionais dos centros especializados. No estudo de Pereira-Silva (2000), a maioria dos genitores de crianças com SD relataram a possibilidade de seu filho(a) vir a se relacionar com pessoas do sexo oposto. Acreditamos que a reação negativa dos genitores e dos profissionais frente a esse tipo de relacionamento seja devido à crença de que essas pessoas são dependentes e não conseguem manejar de forma adequada as situações e, também, devido às possíveis conseqüências desses relacionamentos.

A vida das crianças com síndrome de Down poderia ser bem melhor se sua inserção no contexto sociocultural fosse mais adequada. $\mathrm{O}$ entendimento sobre o tipo de relação que a criança com SD estabelece com seu ambiente, durante seu ciclo de vida, é de grande importância para uma melhor compreensão de aspectos de seu desenvolvimento, bem como de suas interações sociais. Neste particular, a família desempenha um papel fundamental, pois constitui o primeiro universo de relações sociais da criança, podendo proporcionar-lhe um ambiente de crescimento e desenvolvimento ou, ao contrário, um ambiente que venha a dificultar um desenvolvimento mais adequado e saudável.

\section{A importância do ambiente e da família na promo- ção do desenvolvimento de crianças com SD}

Neste tópico, discutimos o papel do ambiente no desenvolvimento da criança, especialmente daquela com deficiência mental e a importância da família na promoção do desenvolvimento desta criança. Os resultados de algumas pesquisas sobre o impacto da criança $\mathrm{DM}^{1}$ no grupo familiar são também destacados.

\section{$O$ papel do ambiente}

Segundo Vygotsky (1994), o ambiente é mutável e dinâmico, exercendo um papel variado no desenvolvimento infantil, dependendo da idade da criança. À medida que esta se desenvolve, seu ambiente também muda, e, conseqüentemente, a sua forma de relação com este meio se altera. Mas, o ambiente a que nos referimos não é apenas o ambiente do lar da criança, mas também outros ambientes que ela começa a freqüentar em cada momento de sua vida, tais como a creche, o jardim-de-infância, a pré-escola etc.. Portanto, o ambiente não deve ser encarado como uma entidade estática e periférica em relação ao desenvolvimento infantil.

A influência do ambiente no desenvolvimento psicológico da criança ocorre, segundo Vygotsky (1994), basicamente através da experiência emocional do sujeito. Em uma experiência emocional estão em relação tanto as características próprias do sujeito, como aquelas do ambiente, mas não é qualquer experiência que se torna relevante para o curso futuro do desenvolvimento da criança. Em decorrência disso, as situações vividas em cada ambiente podem influenciar diferentemente as várias pessoas que ali convivem, uma vez que, cada criança pode ter uma percepção diferente de um evento, experienciando-o de um modo particular e, conseqüentemente, a influência deste sobre o desenvolvimento de cada criança acaba sendo muito peculiar. E é dentro desse contexto que inserimos a nossa criança com SD, a qual apresenta limitações em suas capacidades, porém não está imune às transformações de seu ambiente.

Na concepção de Vygotsky (1994), o ambiente já possui as fontes necessárias para o desenvolvimento 
da criança e, portanto, apresenta uma forma apropriada que deve estar em relação com a forma rudimentar da criança para que o desenvolvimento possa ocorrer sem falhas. A possibilidade de um fracasso em algum aspecto do desenvolvimento decorre da inexistência dessa forma ideal apropriada no ambiente, não havendo assim a interação da criança com a mesma. Mas, não apenas Vygotsky ressalta a importância do ambiente para o desenvolvimento humano. É interessante destacar também a contribuição de Bronfenbrenner (1977, 1979/1996, 1986, 1992), para quem a pessoa é concebida como um todo funcional em que os diversos processos psicológicos - cognitivo, afetivo, emocional, motivacional e social - relacionam-se de forma coordenada um com o outro. Para este autor, o ambiente está estruturado em níveis: macro, exo, meso e microssistema, e cada um influencia e é influenciado pelo outro. Em decorrência da conexão existente entre estes sistemas sociais, uma pessoa no microssistema familiar é influenciada por todos os outros sistemas e é nessa interação que ela se desenvolve.

Esses quatro níveis ${ }^{2}$ de organização do ambiente propostos por Bronfenbrenner (1977, 1979/1996) proporcionam uma idéia ampla de como a criança, incluindo aquela com SD, é influenciada pelos diversos ambientes em que está em contato e, especificamente, pela cultura em que está inserida, pois o desenvolvimento não pode ser entendido sem levar em consideração o processo distal. Este processo envolve as condições históricas, culturais, sociais e, também, ambientais que, por sua vez, estão em relação indireta com a pessoa em desenvolvimento (Tudge, Gray \& Hogan, 1997). Portanto, a criança se desenvolve em um contexto social e humano que lhe dá a possibilidade de interiorizar experiências e construir seu sistema de significados, contribuindo para emergirem novas formas de organização. Nesse sentido, a criança com SD tem a possibilidade de vivenciar novos domínios e de construir-se enquanto pessoa ativa e cidadã dentro de nossa cultura. $\mathrm{O}$ ambiente da família constitui o primeiro universo de socialização da criança, e é o que sofre as mais profundas transformações quando do nascimento de uma criança DM, não só em termos de reações e sentimentos de seus membros, como também de estrutura e funcionamento da família. A literatura (Brito \& Dessen, 1999; Casarin, 1999; Cuskelly \& Dadds, 1992; Leary \& Verth, 1995; Minnes, 1998; Pereira-Silva \& Dessen, 2001; Sloper, Knussen, Turner \& Cunningham, 1991) demonstra uma grande variedade de reações e sentimentos vivenciados pela família, sugerindo que cada uma apresenta recursos diferentes para enfrentar as dificuldades com suas crianças deficientes.

\section{A família e o desenvolvimento da criança}

Segundo Pereira-Silva e Dessen (2001), a família, através das relações estabelecidas entre seus membros, pode proporcionar à criança um ambiente de crescimento e desenvolvimento, especialmente para aquelas com deficiência mental, as quais requerem atenção e cuidados específicos. A família, sendo o primeiro universo de relações da criança, traz em si uma enorme carga emocional que tem uma ação profunda sobre a personalidade de seus membros (Rey \& Martinez, 1989). As relações desenvolvidas neste microssistema possuem características específicas de unicidade e complexidade, constituindo um contexto em desenvolvimento (Kreppner, 1992). Para Kreppner, podemos entender a complexidade das relações familiares por meio da perspectiva da família como um ambiente não compartilhado, cujas relações entre seus membros geram experiências diferenciadas para cada um. Portanto, cada membro da família vivencia, de maneira particular, a chegada de uma criança com deficiência.

O momento do nascimento de uma criança com deficiência é um dos momentos mais difíceis para a família enquanto grupo e para cada um dos seus membros, individualmente, podendo haver alterações nos relacionamentos, especialmente no desenvolvimento do vínculo mãe-bebê (Bolsanello, 1998) e até uma desestruturação inicial, para depois haver uma adaptação mais adequada do grupo (Brito \& Dessen, 1999; Petean, 1995; Taveira, 1995). Especificamente com relação às famílias de crianças com SD, Casarin (1999) afirma que "a família precisa repensar o significado da síndrome de Down para que, reformulando a imagem deformada que possuía, possa construir uma outra, não idealizada, que viabilize seu relacionamento com a pessoa portadora da síndrome" (p. 274). Desta forma, a reorganização familiar pode ficar mais fácil, e, neste caso, o ambiente familiar pode contribuir para o desenvolvimento e crescimento da criança com SD. Contudo, o ambiente pode dificultar essa reorganização interna da família, principalmente porque o nascimento de uma criança, por si só, já traz modificações em diversas dimensões das relações entre os membros de uma família (Dessen, 1997; Kreppner, 1989, 1992).

O equilíbrio da família após o nascimento de uma criança com deficiência é restabelecido de maneira variada, dependendo dos recursos psicológicos utilizados para tal fim. No estudo de Gallimore, Coots, Weisner, Garnier e Guthrie (1996), com famílias que possuíam uma criança pré-escolar com atraso no desenvolvimento, os resultados revelaram que as adaptações destas famílias apresentaram um panorama misto de continuidades e mudanças em seus padrões de interação até a segunda infância da criança. Na adolescência não houve evidências sugerindo que o grau de adaptação diminuísse drasticamente. Assim, 
de acordo com estes autores, em todas as idades, a adaptação da família está relacionada às características da criança, as quais exercem um impacto direto na rotina diária dos membros familiares.

Segundo Zamberlan e Biasoli-Alves (1996), tanto fatores macrossistêmicos - renda familiar, grau de instrução dos pais e profissão, como microssistêmicos - qualidade das interações e relações entre os membros familiares e pessoas próximas, particularmente no que tange às práticas psicossociais de cuidados implementadas nesses contextos, associam-se na promoção de um desenvolvimento adequado e saudável da criança. No entanto, reiteramos o fato de que a família pode exercer um papel tanto estimulador como deletério no desenvolvimento de suas crianças deficientes, já que são inúmeras as variáveis que estão em contínua interação, influenciando a dinâmica e o funcionamento dessas famílias (Pereira-Silva \& Dessen, 2001). É importante aprofundar o conhecimento sobre o funcionamento de famílias de crianças que apresentam algum tipo de deficiência, sobretudo daquelas com SD, para uma melhor compreensão do desenvolvimento dessas crianças e de sua adaptação ao meio. Contudo, o fato de se ter uma criança com SD na família também traz implicações significantes para todos os membros, e a literatura a respeito deste tema é relativamente vasta (Byrne \& Cunnigham, 1985; Dyson, 1997; Gallimore, Coots, Weisner, Garnier \& Guthrie, 1996; Hornby, 1995; Krauss \& Seltzer, 1998; Lamb \& Billings, 1997; Leary \& Verth, 1995; Minnes, 1998; Shapiro, Blacher \& Lopez., 1998; Sloper, Knussen, Turner \& Cunningham, 1991).

\section{O impacto da criança DM na família}

Apesar de haver, no Brasil, uma escassez de estudos na área de relações familiares de crianças que apresentam algum tipo de deficiência (Dessen \& Pereira-Silva, 2000), o efeito dessa criança sobre a sua família tem sido objeto de estudo em outros países há muitas décadas (Byrne \& Cunnigham, 1985; Gallimore e cols., 1996; Krauss \& Seltzer, 1998; Leary \& Verth, 1995; Minnes, 1998). Os aspectos emocionais que envolvem as famílias de crianças DM parecem constituir um dos principais focos de estudo para os pesquisadores. O estresse, por exemplo, é um dos estados emocionais mais comumente investigado, especialmente em mães de crianças com deficiências (Byrne \& Cunningham). Alguns estudos mostram que as mães de crianças DM experienciam mais estresse que os pais e, também, que as mães de crianças com desenvolvimento normal (Hornby, 1995; Lamb \& Billings, 1997; Shapiro e cols., 1998; Sloper e cols., 1991), mas há outros estudos que mostram um nível similar de estresse tanto em mães como em pais de crianças DM em idade escolar (Dyson, 1997). No geral, a literatura indica que o fato de se ter uma criança DM na família pode ser ou não um evento estressante, mas traz consequiências para todos os membros da família em maior ou menor grau, dependendo do funcionamento da mesma.

As famílias com crianças DM têm uma sobrecarga adicional em todos os níveis: social, psicológico, financeiro e, também, nos cuidados com a criança (Ali, Al-Shatti, Khaleque, Rahman, Ali \& Ahmed, 1994, Shapiro e cols., 1998). Essa sobrecarga pode estar relacionada aos sentimentos de ansiedade e incerteza quanto à sobrevivência da criança, ao seu desenvolvimento, ao cuidado a longo prazo e ao próprio impacto desse cuidado sobre a vida pessoal da mãe. Estes fatores podem causar um senso de limitação e restrição, resultante de um compromisso prolongado e crônico de cuidado (Shapiro e cols.). Para os pais, a situação não parece ser tão diferente das mães. Estes, em um estudo com famílias de crianças com SD, relataram sentir mais sobrecarga que os pais de crianças com desenvolvimento normal (Rodrigue, Morgan \& Geffken, 1992). Assim, a sobrecarga parece tornar-se mais um aspecto desencadeante do estresse e/ou de sentimentos vivenciados pelos genitores de crianças DM.

Com relação à depressão e ao ajustamento psicológico, alguns estudos destacam que as mães de crianças DM apresentam taxas mais altas de depressão e enfrentam mais problemas com o ajustamento psicológico que os pais (Glidden \& Floyd, 1997; Negrin \& Cristante, 1996) e, também, que as mães de crianças com desenvolvimento normal (Carr, 1988; Shapiro e cols., 1998). A depressão nessas mães mostrou ser uma reação distinta de outros problemas dos genitores e da família em geral (Glidden \& Floyd). No estudo de Bristol, Gallagher e Schopler (1988), não houve resultados significativos quanto à vivência da depressão entre os dois tipos de famílias. Mas, com relação à instabilidade emocional, alguns estudos concluem que os genitores de crianças DM são mais instáveis emocionalmente que os genitores de crianças com desenvolvimento normal (Ali e cols., 1994; Sloper e cols., 1991). Além destes estados emocionais, podemos observar outros, tais como a mágoa, o sofrimento e um contínuo processo de luto (Leary \& Verth, 1995) e culpa (Ali e cols., 1994).

Embora o impacto da criança DM na família pareça desestruturante, necessitamos corrigir uma visão patológica a respeito das reações dos genitores à sua criança DM. Por exemplo, no estudo de Pereira-Silva (2000), os genitores relataram que se sentiam satisfeitos com os progressos de suas crianças com SD e suas interações foram caracterizadas principalmente pela 'sincronia', 'supervisão' e 'amistosidade', o que demonstra uma adequação entre os comportamentos dos genitores e os de suas crianças. Assim, podemos 
perceber que as diferenças encontradas nas pesquisas quanto às reações de mães de crianças com e sem DM e entre mães e pais de crianças DM, podem sugerir que as mães continuam sendo mais fortemente afetadas pelas obrigações de cuidados associados com a criança DM. Por outro lado, não se tem clareza se os altos níveis de sintomatologia emocional e física dos genitores estão relacionados à presença dessa criança ou a uma rede de variáveis mediadoras ou, ainda, às diferenças entre homens e mulheres na grande população (Shapiro e cols., 1998).

\section{CONSIDERAÇÕES FINAIS}

É fundamental que pesquisadores e profissionais voltem a sua atenção para a compreensão da dinâmica de funcionamento de famílias de crianças com SD, uma vez que a família constitui o primeiro agente de socialização da criança e é a mediadora das relações desta com seus diversos ambientes. Portanto, conhecer como se processam as interações entre a criança com $\mathrm{SD}$ e seus genitores e irmãos possibilita compreender as relações futuras desta criança com seus companheiros, bem como a sua inserção nos diversos contextos socioculturais. Em um levantamento da produção cientifica na área de deficiência mental e relações familiares efetuado por Dessen e Pereira-Silva (2000), $33,7 \%$ dos artigos focalizavam o tema da 'família e sua criança DM', com destaques para o estresse decorrente de se ter uma criança DM e o seu efeito no funcionamento da família, em se tratando de pesquisas estrangeiras. No Brasil, o foco das pesquisas era sobre as atitudes/percepções e estados emocionais dos genitores frente à sua criança DM. Portanto, há necessidade não só de aumentar o número de pesquisas, mas também de diversificar os temas investigados.

Pereira-Silva e Dessen (2001) relataram que os genitores de crianças com SD têm recebido pouca informação e orientação a respeito da síndrome e de suas consequiências para o desenvolvimento da criança. É evidente que as famílias precisam ser orientadas quanto à maneira mais adequada de estimular o desenvolvimento de suas crianças e cuidar de sua saúde física mas, elas devem, sobretudo, ser orientadas sobre como estabelecer interações e relações saudáveis com a sua criança com SD, mantendo o equilíbrio do grupo familiar. Neste contexto, as políticas públicas desempenham um papel fundamental, particularmente no que se refere às famílias de baixa renda, uma vez que o gasto com profissionais e com atendimento especializado torna-se oneroso.

No Brasil, o Ministério da Educação (MEC) é o responsável pela organização e administração do ensino especial, juntamente com a participação estadual e municipal (MEC/SEESP, 1994). O ensino especial é, portanto, regulamentado por lei, mas nem todos os
Estados e Municípios brasileiros contam com todos os tipos de atendimentos que ele pode oferecer. O Programa de Estimulação Precoce, por exemplo, faz parte dos diversos tipos de atendimentos planejados pelo $\mathrm{MEC}$, oferecido às crianças de zero a três anos de idade com atraso no desenvolvimento ou com algum tipo de deficiência, seja física, mental, visual, auditiva ou múltipla. Esse programa tem, em sua essência, um papel preventivo, buscando intervir nas diversas áreas do desenvolvimento infantil: motora, cognitiva, sensório-perceptiva, socioafetiva e da linguagem (MEC/ SEESP, 1995). Assim, dada a necessidade desse tipo de atendimento às crianças com deficiências, desde o seu nascimento, entendemos ser de fundamental importância mais investimentos nessa área, atingindo mais Estados e Municípios brasileiros e, com isto, mais famílias poderiam ser melhor atendidas e preparadas para desenvolver interações mais saudáveis com suas crianças.

Epps e Jackson (2000) propõem que a intervenção deva ocorrer em nível das interações e relações familiares, para que se obtenha resultados mais satisfatórios, tanto para as crianças como para as famílias. Além dessa premissa, os autores ressaltam mais quatro fatores que devem, ou deveriam, constar das linhas gerais dos programas de educação familiar: (a) focalizar a família como um todo, (b) enfatizar a competência e a capacidade de recuperação da criança e da família, (c) incluir a comunidade e as parcerias com diversos segmentos do sistema social e (d) ser dirigido empiricamente. A idéia de que a intervenção deva ocorrer em nível das interações e relações familiares é compartilhada também por Spiker (1990), que ressalta a importância de focalizar a interação genitores-criança e a responsividade dos genitores, que são fatores preponderantes para o sucesso de programas voltados para crianças com SD. Assim, parece não haver dúvidas de que a eficácia dos programas de "educação familiar" depende de uma visão sistêmica da família, considerada como o foco da intervenção, particularmente na dimensão das relações entre os seus membros.

\section{REFERÊNCIAS}

Ali, M. R.; Al-Shatti, A.; Khaleque, A.; Rahman, A.; Ali, S. M. \& Ahmed, R. U. (1994). Parents of mentally retarded children: Personality characteristics and psychological problems. Social Behavior and Personality, 22, 41-52.

Bolsanello, M. A. (1998). Interação mãe-filho portador de deficiência: concepções e modo de atuação dos profissionais em estimulação precoce. Tese de Doutorado, Universidade de São Paulo, São Paulo.

Brito, A. M. W. \& Dessen, M. A. (1999). Crianças surdas e suas famílias: Um panorama geral. Psicologia: Reflexão e Crítica, 12, 429-445. 
Bristol, M. M.; Gallagher, J. J. \& Schopler, E. (1988). Mothers and fathers of young developmentally disabled and nondisabled boys: Adaptation and spousal support. Developmental Psychology, 24, 441-451.

Bronfenbrenner, U. (1977). Toward an experimental ecology of human development. American Psychologist, 32, 513-531.

Bronfenbrenner, U. (1986). Ecology of the family as a context for human development: Research perspectives. Developmental Psychology, 22, 723-742.

Bronfenbrenner, U. (1992). Ecological systems theory. Em R. Vasta (Org.), Six theories of child development (p. 187-243). London: Jessica Kingsley Publishers.

Bronfenbrenner, U. (1996). A ecologia do desenvolvimento humano: experimentos naturais e planejados. (M. A. Veríssimo, Trad.) Porto Alegre: Artes Médicas. (Trabalho originalmente publicado em 1979).

Brunoni, D. (1999). Aspectos epidemiológicos e genéticos. Em J. S. Schwartzman (Org.), Síndrome de Down (p. 32-43). São Paulo: Mackenzie.

Byrne, E. A. \& Cunningham, C. C. (1985). The effects of mentally handicapped children on families. A conceptual review. Journal of Child Psychology and Psychiatry, 25, 847-864.

Carr, J. (1988). Six weeks to twenty-one years old: A longitudinal study of children with Down's syndrome and their families. Journal of Child Psychology and Psychiatry, 29, 407-431.

Carr, J. (1994). Annotation: Long term outcome for people with Down's syndrome. Journal of Child Psychology and Psychiatry, 35, 425-439.

Casarin, S. (1999). Aspectos psicológicos na síndrome de Down. Em J. S. Schwartzman (Org.), Síndrome de Down (p. 263-285). São Paulo: Mackenzie.

Cuskelly, M. \& Dadds, M. (1992). Behavioural problems in children with Down's syndrome and their siblings. Journal of Child Psychology and Psychiatry, 33, 749. 761.

Dessen, M. A. (1997). Desenvolvimento familiar: transição de um sistema triádico para poliádico. Temas em Psicologia, 3, 51-61.

Dessen, M. A. \& Pereira-Silva, N. L. (2000). Deficiência mental e família: uma análise da produção científica. Cadernos de Psicologia e Educação Paidéia, 10, 12-23.

Dyson, L. L. (1997). Fathers and mothers of school-age children with developmental disabilities: Parental stress, family functioning, and social support. American Journal on Mental Retardation, 102, 267-279.

Epps, S. \& Jackson, B. J. (2000). Empowered families, successful children. Washington, DC: American Psychological Association.

Gallimore, R.; Coots, J.; Weisner, T.; Garnier, H. \& Guthrie, D. (1996). Family responses to children with early developmental delays II: Accommodation intensity and activity in early and middle childhood. American Journal on Mental Retardation, 101, 215-232.

Ganiban, J.; Wagner, S. \& Cicchetti, D. (1990). Temperament and Down syndrome. Em D. Cicchetti \& M. Beeghly (Orgs.), Children with Down syndrome: A developmental perspective (p. 63-100). Cambridge: Cambridge University Press.
Glidden, L. M. \& Floyd, F. J. (1997). Disaggregating parental depression and family stress in assessing families of children with developmental disabilities: A multisample analysis. American Journal on Mental Retardation, 102, 250-266.

Hornby, G. (1995). Effects on fathers of children with Down syndrome. Journal of Child and Family Studies, 4, 239-255.

Krauss, M. W. \& Seltzer, M. M. (1998). Life course perspectives in mental retardation research: The case of family caregiving. Em J. A. Burack; R. M. Hodapp \& E. Zigler (Orgs.), Handbook of mental retardation and development (p. 504-520). Cambridge: Cambridge University Press.

Kreppner, K. (1989). The interplay between individual and family development: Some results from a 7 - year longitudinal study. Em M. A. Luszcz \& T. Nettelbeck (Orgs.), Psychological development: Perspectives across the life-span (p. 25-36). North-Holland: Elsevier Science Publishers.

Kreppner, K. (1992). Development in a developing context: Rethinking the family's role for children's development. Em L. T. Winegar \& J. Valsiner (Orgs.), Children's development within social context (p. 161-179). Hillsdale: Lawrence Erlbaum.

Lamb, M. E. \& Billings, L. A. L. (1997). Fathers of children with special needs. Em M. E. Lamb (Org.), The role of the father in child development (p. 179-190). New York: Wiley.

Leary, P. M. \& Verth, F. (1995). The effects of a mentally retarded child on family functioning in a third world community. Early Child Development and Care, 109, 83-88.

Lemanek, K. L.; Stone, W. L. \& Fishel, P. T. (1993). Parent-child interactions in handicapped preschoolers: The relation between parent behaviors and compliance. Journal of Clinical Child Psychology, 22, 68-77.

Ministério da Educação do Brasil [MEC] (1994). Educação especial no Brasil. Brasília, DF: MEC/SEESP .

Ministério da Educação do Brasil [MEC] (1995). Diretrizes sobre estimulação precoce: o portador de necessidades educativas especiais. Brasília, DF: MEC/SEESP.

Minnes, P. (1998). Mental retardation: The impact upon the family. Em J. A. Burack, R. M. Hodapp \& E. Zigler (Orgs.), Handbook of mental retardation and development (p. 693-712). Cambridge: Cambridge University Press.

Negrin, N. S. \& Cristante, F. (1996). Resources and stress in parents with a mentally retarded child: A quantitative approach. Em M. Cusinato (Org.), Research on family: Resources and needs across the world (p. 493-506). Milão: LED - Edicioni Universitarie.

Pereira-Silva, N. L. (2000). Crianças pré-escolares com síndrome de Down e suas interações familiares. Dissertação de Mestrado, Universidade de Brasília, Brasília.

Pereira-Silva, N. L. \& Dessen, M. A. (2001). Deficiência mental e família: implicações para o desenvolvimento da criança. Psicologia: Teoria e Pesquisa, 17, 133-141.

Petean, E. B. L. (1995). Avaliação qualitativa dos aspectos psicológicos do aconselhamento genético através do estudo prospectivo do atendimento das famílias. Tese de 
Doutorado, Faculdade de Ciências Médicas da UNICAMP, Campinas.

Rey, F. G. \& Martinez, A. M. (1989). La personalidad: su educación y desarrollo. La Habana: Editorial Pueblo y Educación.

Rodrigue, J. R.; Morgan, S. B. \& Geffken, G. R. (1992). Psychosocial adaptation of fathers of children with autism, Down syndrome and normal development. Journal of Autism and Development Disorders, 22, 249-263.

Schwartzman, J. S. (1999a). Histórico. Em J. S. Schwartzman (Org.), Síndrome de Down (p. 3-15). São Paulo: Mackenzie.

Schwartzman, J. S. (1999b). Generalidades. Em J. S. Schwartzman (Org.), Síndrome de Down (p. 16-31). São Paulo: Mackenzie.

Schwartzman, J. S. (1999c). O sistema nervoso na síndrome de Down. Em J. S. Schwartzman (Org.), Síndrome de Down (p. 44-81). São Paulo: Mackenzie.

Shapiro, J.; Blacher, J. \& Lopez, S. R. (1998). Maternal reactions to children with mental retardation. Em J. A. Burack; R. M. Hodapp \& E. Zigler (Orgs.), Handbook of mental retardation and development (p. 606-636). Cambridge: Cambridge University Press.

Sloper, P.; Knussen, C.; Turner, S. \& Cunningham, C. (1991). Factors related to stress and satisfaction with life in families of children with Down's syndrome. Journal of Child Psychology and Psychiatry, 32, 655-676.

Spiker, D. (1990). Early intervention from a developmental perspective. Em D. Cicchetti \& M. Beeghly (Orgs.), Children with Down syndrome: A developmental perspective (p. 424-448). Cambridge: Cambridge University Press.
Taveira, R. M. T. (1995). Privação auditiva precoce em crianças portadoras da síndrome de Down e suas implicações para o desenvolvimento da linguagem. Dissertação de Mestrado, Universidade de Brasília, Brasília.

Tristão, R. M. \& Feitosa, M. A. G. (1998). Linguagem na síndrome de Down. Psicologia: Teoria e Pesquisa, 14, 127-137.

Tudge, J., Gray, J. T. \& Hogan, D. M. (1997). Ecological perspectives in human development: A comparison of Gibson and Bronfenbrenner. Em J. Tudge; M. J. Shanahan \& J. Valsiner (Orgs.), Comparisons in human development: Understanding time and context (p. 72-105). Cambridge: Cambridge University Press.

Turnbull, A. P. \& Ruef, M. (1996). Family perspectives on problem behavior. Mental Retardation, 34, 280-293.

Vygotsky, L. (1994). The problem of the environment. Em R. Van der Veer \& J. Valsiner (Orgs.), The Vygotsky Reader (p. 338-354). Oxford, UK: Basil Blackwell.

Zamberlan, M. A. T. \& Biasoli-Alves, Z. M. M. (1996). Interações familiares: teoria, pesquisa e subsídios à intervenção. Londrina: Editora da UEL.

\section{Notas:}

${ }^{1}$ Usamos a sigla DM para referir-nos à criança com deficiência mental.

${ }^{2}$ Para conceituação e caracterização dos níveis e/ou detalhes sobre o modelo bioecológico proposto por Bronfenbrenner consultar: Bronfenbrenner, U. (1999). Environments in developmental perspective: theoretical and operational models. Em S. L. Friedman \& T. D. Wachs (Orgs.), Measuring environment across the life span: emerging methods and concepts (p. 3-28). Washington D.C: American Psychological Association. Bronfenbrenner, U. \& Morris, P. A. (1998). The ecology of developmental processes. Em W. Damon \& R. M. Lerner (Orgs.), Handbook of child pshycology: v. 1: Theoretical models of human development (p. 993-1028). NY: John Wiley \& Sons.

\section{Sobre as autoras}

Nara Liana Pereira Silva: Mestre e doutoranda em Psicologia pela Universidade de Brasília - UnB, na área de Desenvolvimento Humano. Atualmente, participa de pesquisas vinculadas ao Laboratório de Desenvolvimento Familiar do Instituto de Psicologia da UnB. É, também, psicóloga da Fundação Educacional do Distrito Federal, com atuação voltada para o Ensino Especial.

Endereço para correspondência: Universidade de Brasília - UnB, Instituto de Psicologia - Laboratório de Desenvolvimento Familiar. Campus Universitário. Asa Norte, Brasília, DF - CEP 70910-900. E-mail: liana@unb.br

Maria Auxiliadora Dessen: Doutora em Psicologia pela Universidade de São Paulo, com pós-doutoramento pela Universidade de Lancaster, Inglaterra. É professora do Instituto de Psicologia da Universidade de Brasília e coordenadora do Laboratório de Desenvolvimento Familiar - IP/UnB. Atualmente desenvolve atividades de pesquisa no Instituto Max Planck para o Desenvolvimento Humano, em Berlim. 